\title{
Acute Pancreatitis in COVID-19 Patients: Two Case Reports and Review of Literature
}

Sreenivas Reddy Madhurantakam ${ }^{1}$, Padmakumar V Arayamparambil ${ }^{2}$, Garud Suresh Chandan ${ }^{3}$, Pooja Prathapan Sarada ${ }^{4 *}$ ${ }^{\mathrm{T}}$ Senior Registrar, Department of Critical Care Medicine, Fortis Hospital Bannerghatta Road, 154/9, Opp. IIM-B, Bangalore-560076, Karnataka, India

${ }^{2}$ Director \& Head of the Department, Department of Critical Care Medicine, Fortis Hospital Bannerghatta Road, 154/9, Opp. IIM-B, Bangalore-560076, Karnataka, India

${ }^{3}$ Senior Consultant, Department of Critical Care Medicine, Fortis Hospital Bannerghatta Road, 154/9, Opp. IIM-B, Bangalore-560076, Karnataka, India

${ }^{4}$ Consultant, Department of Critical Care Medicine, Fortis Hospital Bannerghatta Road, 154/9, Opp. IIM-B, Bangalore-560076, Karnataka, India

\section{Article History}

Received: 03.12 .2020

Accepted: 16.12 .2020

Published: 18.12 .2020

Journal homepage:

https://www.easpublisher.com

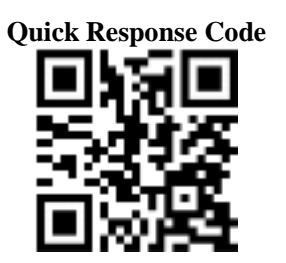

Abstract: Coronavirus disease 2019 (COVID-19) is primarily a respiratory tract disease but involves other organ systems too, especially gastrointestinal system. Acute pancreatitis is one of the manifestations of COVID-19, as pancreatic gland also expresses the angiotensinconverting enzyme 2 receptors. We present two cases, where none of them presented with symptoms of pancreatitis on admission. Our first patient presented with severe acute respiratory distress syndrome secondary to COVID-19 and during her stay necrotizing pancreatitis was incidentally detected on CT scan. Our second patient presented as an acute fatty liver of pregnancy and was incidentally found to be COVID-19 positive and later developed symptoms of acute pancreatitis.

Keywords: COVID-19, Acute Pancreatitis, lipase, Necrotizing pancreatitis, SARS-CoV-2.

Copyright (C) 2020 The Author(s): This is an open-access article distributed under the terms of the Creative Commons Attribution 4.0 International License (CC BY-NC 4.0) which permits unrestricted use, distribution, and reproduction in any medium for non-commercial use provided the original author and source are credited.

\section{INTRODUCTION}

The Severe Acute Respiratory Syndrome Coronavirus-2 (SARS CoV-2) pandemic has created havoc across the globe. It's a multisystemic disease mainly involving the upper respiratory tract system. The extrapulmonary manifestations are still not very well understood. The gastrointestinal (GI) manifestations of the COVID-19 infection include anorexia, nausea, vomiting, abdominal pain, and diarrhoea. Pancreatic gland expresses angiotensin-converting enzyme 2 (ACE-2), the proven receptor for SARS-CoV-2 cell binding. The symptoms of pancreatitis in COVID-19 patients vary from asymptomatic abnormal pancreatic enzyme elevation to necrotizing pancreatitis. However, pancreatic involvement in patients with COVID-19 is rarely reported. Here, we report two cases of acute pancreatitis with COVID-19.

\section{Case Report-1}

A 48 year old, female, who was a known case of diabetes mellitus, hypertension, hypothyroidism, end stage renal disease on maintenance haemodialysis through a long term dialysis catheter and previously acquired Hepatitis $\mathrm{C}$ infection, presented to us with complaints of fever, giddiness and weakness for 3 days. She had also missed her regular dialysis due to fever.

Initial evaluation revealed a temperature of $101^{\circ} \mathrm{F}$, blood oxygen saturation ( $\left.\mathrm{SpO} 2\right)$ of $89 \%$ on 10 liter/minute of oxygen and arterial blood gas (ABG) analysis showed metabolic acidosis. Patient was tested positive for COVID-19. After the initial Non-invasive ventilation trial, she was intubated and dialysed for severe metabolic acidosis. She became hemodynamically unstable requiring vasopressors. Patient was managed with steroids, anticoagulation and prone ventilation for severe acute respiratory distress syndrome (ARDS). Antibiotics were started for suspected central line - associated bloodstream infections (CLABSI). Initial blood cultures grew Candida tropicalis, endotracheal secretion culture grew Klebsiella pneumonia (ESBL+ Carbapenemase producing). Long term dialysis catheter was removed. 
As the patient's haemodynamics and oxygenation improved, she was tracheostomized and gradual weaning from the ventilator was started in the $3^{\text {rd }}$ week of admission. She developed persistent non infective diarrhoea during her stay. After 4 weeks of admission, the patient was tested negative for COVID19 with demonstrable IgG antibody. Patient's computed tomography (CT) abdomen showed evidence of acute/subacute necrotizing pancreatitis with peripancreatic walled-off collection (Figure-1). In view of this her serum lipase and triglycerides were sent which was 69 Units/Liter and $3.22 \mathrm{mmol} / \mathrm{L}$ respectively.

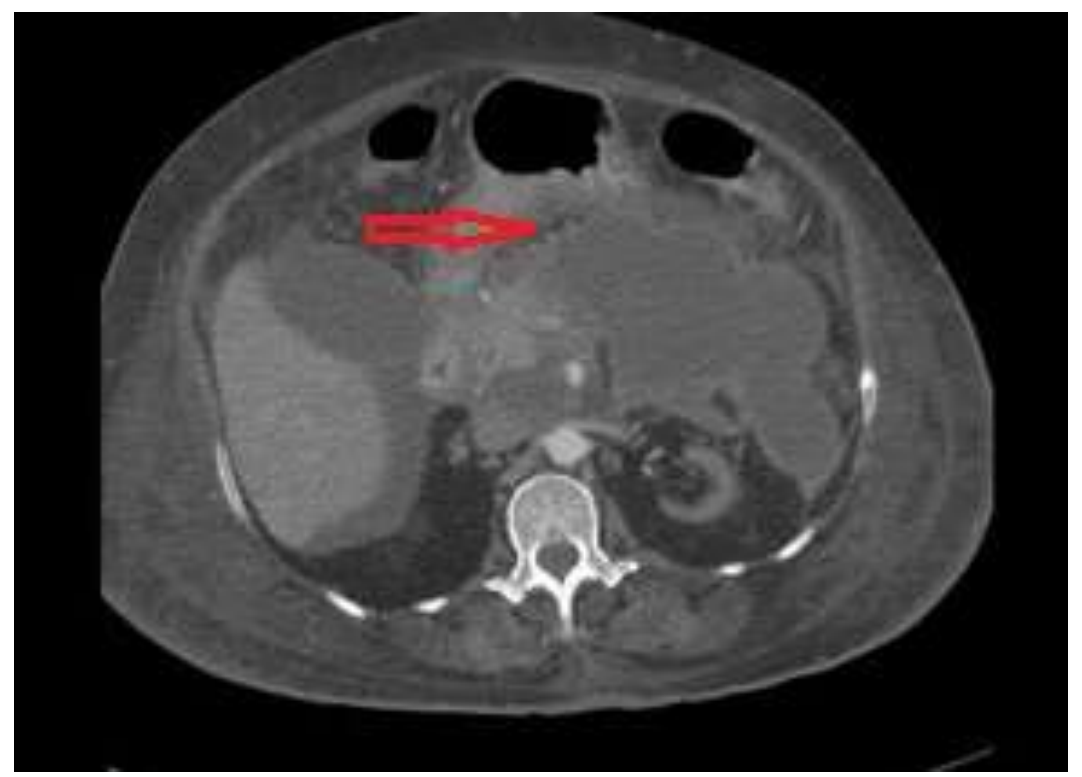

Fig-1: CT image showing acute necrotizing pancreatitis (arrow) with peripancreatic walled-off collection

\section{Case Report-2}

A 27 year old primigravida at 37 weeks of gestation was admitted with acute fatty liver of pregnancy, acute kidney injury and was incidentally detected to be COVID-19 positive. Preoperative ultrasound sonography (USG) abdomen was found to be normal. She underwent an emergency caesarean section for obstetric indication. Blood and blood products were transfused for coagulopathy. She remained stable for the next $36 \mathrm{hrs}$. On post operative day - 3, she complained of abdominal discomfort and distention. USG abdomen was done that showed edematous pancreas. Her serum amylase (363U/L) and lipase (3885U/L) were found to be elevated. Because of progressive drowsiness and worsening coagulopathy, she was intubated. CT brain showed normal study. CT abdomen showed acute interstitial edematous pancreatitis with no evidence of gallbladder sludge, stone or dilatation of main pancreatic duct or any biliary dilatation (Figure-2). She progressively became more unstable with worsening acute kidney injury associated with lactic acidosis requiring hemodialysis. Patient was found to have significant hypocalcemia with QTc $>700$ milliseconds requiring continuous calcium gluconate infusion. Her sensorium remained poor in spite of correcting metabolic parameters. Patient succumbed to fulminant cerebral edema on day 8 of admission. 


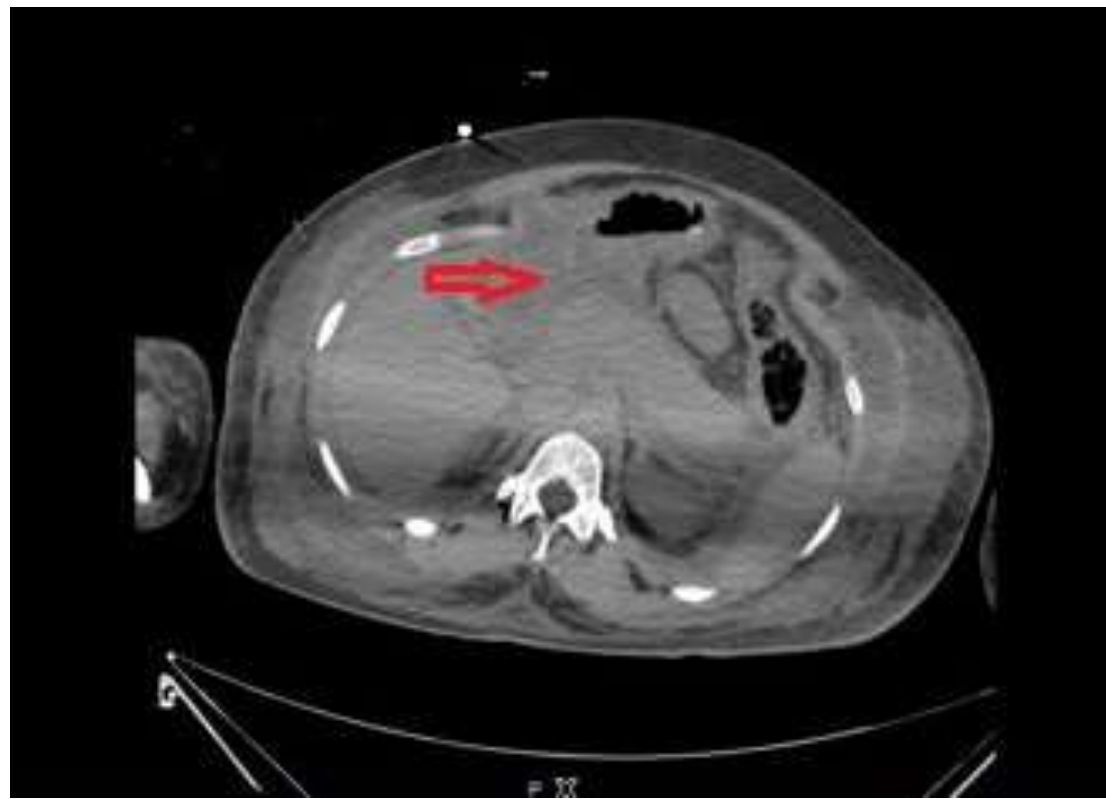

Fig-2: CT image showing acute interstitial edematous pancreatitis (arrow)

\section{DISCUSSION}

COVID-19 is a multisystemic condition caused by coronavirus 2 with manifestations ranging from mild upper respiratory symptoms to cytokine storm causing severe ARDS. COVID-19 has been widely studied as a lung pathogen. However, GI association with COVID- 19 has also been reported in recent studies. Studies have shown that up to $50 \%$ of COVID-19 patients develop GI symptoms of nausea, vomiting, diarrhoea and abdominal pain [1]. In a recent study, $11.4 \%$ of the patients exhibited at least one of the GI symptoms involving nausea, vomiting, or diarrhoea [2].

The pathogenesis of acute pancreatitis is multifactorial. Gallstones and alcohol being the leading cause, viruses such as mumps, Coxsackie B, measles, Epstein-Barr and hepatitis A, B, and E can also cause acute pancreatitis. There has also been few reported associations between $\mathrm{H} 1 \mathrm{~N} 1$ influenza and acute pancreatitis [3-5].

Up to 10 percent of acute pancreatitis is thought to have an infectious aetiology through an immune-mediated inflammatory response, most notably mumps and Coxsackie $\mathrm{B}$ viruses $[6,7]$.

Although viral acute pancreatitis has been described in other viral infections, pancreatic involvement in SARS-CoV-2 infection is still poorly defined. We are reporting two cases of acute pancreatitis in patients with SARS-CoV-2 infection.

ACE2 receptors does not only express in the lung alveolar type-2 (AT-2) cells but also manifests in the esophagus, small intestine, large intestine, and pancreatic islet cells [8]. Glycosylated-spike (S) protein, which is one of the structural proteins encoded by the coronavirus genome, binds to ACE2 receptor protein located on the host cell surface membrane and mediates the host cell invasion $[9,10]$. High expression of ACE2 receptors in the pancreatic islet cells can cause cell damage due to COVID-19, resulting in acute pancreatitis. Direct cytopathic effects of COVID-19 or immune-mediated and indirect systemic inflammatory response could be the mechanism of pancreatic injury [11-13].

Patient-1 developed unexplained diarrhoea 2 to 3 weeks after admission and CT abdomen incidentally picked up acute pancreatitis with already established peripancreatic septated collections. In the initial weeks of admission, sedation and paralysis for severe ARDS probably masked the symptoms of acute pancreatitis. The non-specific symptoms of the patient were correlated retrospectively after CT imaging. Patient -2 developed signs and symptoms of pancreatitis with symptoms like diffuse abdominal pain and symptomatic hypocalcemia.

Since the emergence of the SARS CoV- 2 pandemic in December 2019, a few studies have reported a potential pancreatic injury in association with SARS-CoV-2 infection attributed to either a direct cytopathic effect of the virus or subsequent to the associated systemic inflammatory response.

Safaa Saeed Al Mazrouei, Kataria et al., [5], in their case reports, suggested the possibility of direct causal relation between COVID-19 infection and acute pancreatitis. Antunes Meireles et al., [14] reported acalculous acute pancreatitis in a COVID-19 patient and concluded acute pancreatitis may be a manifestation of SARS-CoV-2 infection. Anand et al., [6] have reported the case of a 59-year-old female patient who was diagnosed with acute pancreatitis 
based on typical abdominal pain and imaging findings 10 days after positive PCR-confirmed SARS-CoV-2 infection.

Wang F, Wang H, Fan J et al., [15], in their study concluded that, mild pancreatic injury patterns in patients with COVID-19 pneumonia, may be related to direct viral involvement of the pancreas or from secondary enzyme abnormalities in the context of severe illness without substantial pancreatic injury.

\section{CONCLUSION}

SARS-CoV-2 has complex presentations because of multisystem involvement including the GI system. COVID-19 induced acute pancreatitis is a rare cause, and other common causes of acute pancreatitis must be ruled out. Our case reports, highlights the importance of considering SARS-CoV-2 as a new aetiological agent of acute viral pancreatitis. This article not only serves to exemplify the association between SARS CoV-2 and acute pancreatitis but also highlights the need for a high index of clinical suspicion for acute pancreatitis in patients with significant inflammatory response and abdominal pain with a COVID positive status. Further studies and larger series are needed to establish the clinical significance of pancreatic injury in COVID-19 patients.

\section{REFERENCES}

1. Weston, S., \& Frieman, M. B. (2020). COVID-19: knowns, unknowns, and questions. Msphere, 5(2):e00203-20.

2. Zhang, Y., Chen, C., \& Zhu, S. (2020). Isolation of 2019-nCoV from a stool specimen of a laboratoryconfirmed case of the coronavirus disease 2019 (COVID-19). China CDC Weekly. 2(8):123-124.

3. Agzarian, A. E., \& Agzarian, A. Y. (2016). Influenza A as a cause of acute pancreatitis: A Case Report. Proceedings of UCLA Healthcare, 20.

4. Habib, A., Jain, A., Singh, B., \& Jamshed, N. (2016). H1N1 influenza presenting as severe acute pancreatitis and multiorgan dysfunction. The American journal of emergency medicine, 34(9), 1911-e1.
5. Al Mazrouei, S. S., Saeed, G. A., \& Hilali, A. (2020). COVID-19-associated acute pancreatitis: A rare cause of acute abdomen. Radiology Case Reports. 15(9):1601-1603.

6. Anand, E. R., Major, C., Pickering, O., \& Nelson, M. (2020). Acute pancreatitis in a COVID-19 patient. The British Journal of Surgery, 107(7), e182.

7. Rawla, P., Bandaru, S. S., \& Vellipuram, A. R. (2017). Review of infectious etiology of acute pancreatitis. Gastroenterology Research, 10(3), 153-158.

8. Buruk, K., \& Ozlu, T. (2020). New coronavirus: SARS-COV-2. Mucosa. 3:1-4.

9. Anthony, S. J., Johnson, C. K., Greig, D. J., Kramer, S., Che, X., Wells, H., ... \& Karesh, W. (2017). Global patterns in coronavirus diversity. Virus evolution, 3(1).

10. Su, S., Wong, G., Shi, W., Liu, J., Lai, A. C., Zhou, J., ... \& Gao, G. F. (2016). Epidemiology, genetic recombination, and pathogenesis of coronaviruses. Trends in microbiology, 24(6), 490502.

11. Jin, X., Lian, J. S., Hu, J. H., Gao, J., Zheng, L., Zhang, Y. M., ... \& Yu, G. D. (2020). Epidemiological, clinical and virological characteristics of 74 cases of coronavirus-infected disease 2019 (COVID-19) with gastrointestinal symptoms. Gut, 69(6), 1002-1009.

12. Chen, L., \& Li, J. (2020). Clinical features of COVID-19-related liver functional abnormality. Clin. Gastroenterol. Hepatol, 18(7), 1561-1566.

13. Kataria, S., Sharif, A., Rehman, A. U., Ahmed, Z., \& Hanan, A. (2020). COVID-19 induced acute pancreatitis: a case report and literature review. Cureus, 12(7):e9169.

14. Meireles, P. A., Bessa, F., Gaspar, P., Parreira, I., Silva, V. D., Mota, C., \& Alvoeiro, L. (2020). Acalculous Acute Pancreatitis in a COVID-19 Patient. European Journal of Case Reports in Internal Medicine, 7(6): 1710.

15. Wang, F., Wang, H., Fan, J., Zhang, Y., Wang, H., \& Zhao, Q. (2020). Pancreatic injury patterns in patients with COVID-19 pneumonia. Gastroenterology. 159(1):367-370. 